\title{
MOLECULAR DYNAMICS OF CHEMICAL REACTIONS
}

\author{
D. R. HERSCHBACH \\ Department of Chemistry, Harvard University, Cambridge, Massachusetts, USA
}

\begin{abstract}
Molecular beam and spectroscopic techniques allow detailed study of many dynamical properties of single reactive collisions. The chemical scope of these methods is now very wide and includes certain unimolecular and termolecular reactions as well as bimolecular reactions and energy transfer processes. Results for more than 50 families of $A+B C \rightarrow A B+C$ atom transfer reactions reveal simple impulsive and persistent complex regimes that correlate with electronic structure. Recent work has found examples of $A B+C D \rightarrow A D+B C$ and $A B+C D+E F \rightarrow$ $A F+B C+D E$ reactions that require exchange of two or three pairs of bonds in a single collision event yet proceed with practically no activation energy. Processes akin to liquid phase reactions are also becoming accessible to dynamical studies using beams of van der Waals polymers or solvation clusters.
\end{abstract}

The study of the intimate mechanics of reaction and energy transfer in individual molecular collisions has become one of the most active frontiers of chemical physics. The main experimental approach is molecular beam scattering. ${ }^{1-4}$ This involves forming the reagent molecules into two collimated beams, each so dilute that collisions within them are negligible. The two beams intersect in a vacuum and the direction and energy of the product molecules recoiling from the collision zone are measured. A host of reactions can now be studied readily in this way, by virtue of an extremely sensitive mass spectrometric detector and the use of supersonic nozzles which produce beams with greatly enhanced intensity and with collision energies much higher than provided by ordinary thermal sources. The coupling of spectroscopic techniques with molecular beams has provided further advances in selectivity and sensitivity, particularly by use of intense laser sources.

The reaction properties now accessible include the disposal of energy among translation, rotation, and vibration of the product molecules; angles of product recoil; angular momentum and its orientation in space; and variation of reaction yield with impact energy, closeness of collision, rotational orientation or vibrational excitation of the target molecule. These experiments have stimulated many theoretical developments, ${ }^{5}$ including very useful diagnostic procedures based on information theory, ${ }^{6}$ a variety of insightful mechanical models, ${ }^{7}$ and the burgeoning field of "computer experiments" in which enormous numbers of collision trajectories are calculated for various postulated forces and compared with model calculations and laboratory data. ${ }^{8}$

In this paper we illustrate some of the molecular beam experimental methods and results for a few prototype reactions. We give a heuristic discussion of two favorite reactions which exemplify the contrasting regimes of impulsive and persistent complex dynamics in atom transfer processes,

$$
A+B C \rightarrow A B+C .
$$

Single-collision data for more than fifty families of such reactions have now been obtained and many other aspects are treated in more comprehensive reviews. ${ }^{1-4}$ In particular, we do not discuss here the very fruitful work on ion-molecule reaction dynamics. ${ }^{9}$ Our main emphasis is on recent studies dealing with remarkably facile exchange reactions involving two or three pairs of bonds,

$$
\begin{gathered}
A B+C D \rightarrow A D+B C \\
A B+C D+E F \rightarrow A F+B C+D E .
\end{gathered}
$$

These results have prompted electronic structure calculations which suggest that the isotopic exchange reaction of molecular hydrogen and deuterium, long presumed to be bimolecular, is in fact termolecular. Finally, we briefly consider new work on reactions of van der Waals polymers and solvation clusters, which offer prospects for dynamical studies of processes akin to liquid phase reactions.

\section{EXPERIMENTAL PERSPECTIVES}

Figure 1 shows typical reactive scattering data for three systems obtained from a crossed-beam apparatus with mass spectrometric detector. ${ }^{10}$ The detector unit is mounted on a rotatable "lid" which allows it to scan a wide range of scattering angles in the plane of the reactant beams. About $0.1 \%$ of the incoming molecules are ionized. Velocity analysis is performed by installing a slotted disk "chopper" which is rotated at high speeds to pulse the beam of scattered product molecules and thus allow measurement of the time of flight. A small computer converts the flight times to a velocity distribution of the product, subtracts the background counts, and prints out the data. The scattering angles indicated are measured from the direction of the reactant atom beam (designated as $0^{\circ}$ ) toward the reactant molecule beam (designated as $90^{\circ}$ ). At the peak of the product distribution, the signal is only about $10-100$ counts per second. The total counting time required to collect the data shown was about five hours for each reaction. Without going into details of the analysis, we can see directly from Fig. 1 that the three reactions display qualitatively different dynamics. In the $\mathrm{D}+\mathrm{Br}_{2}$ reaction, the $\mathrm{DBr}$ product emerges with high velocity and the intensity increases at wide scattering angles; this is evidence for repulsion in the exit valley of the potential surface. In the $\mathrm{Cl}+\mathrm{Br}_{2}$ reaction, the $\mathrm{BrCl}$ emerges with rather low velocity and its intensity distribution strongly favors low angles; this is evidence for an attractive interaction. In the reaction of chlorine atoms with vinyl bromide, the product distribution is approximately uniform with angle; the detailed analysis shows this system in fact displays statistical behavior 


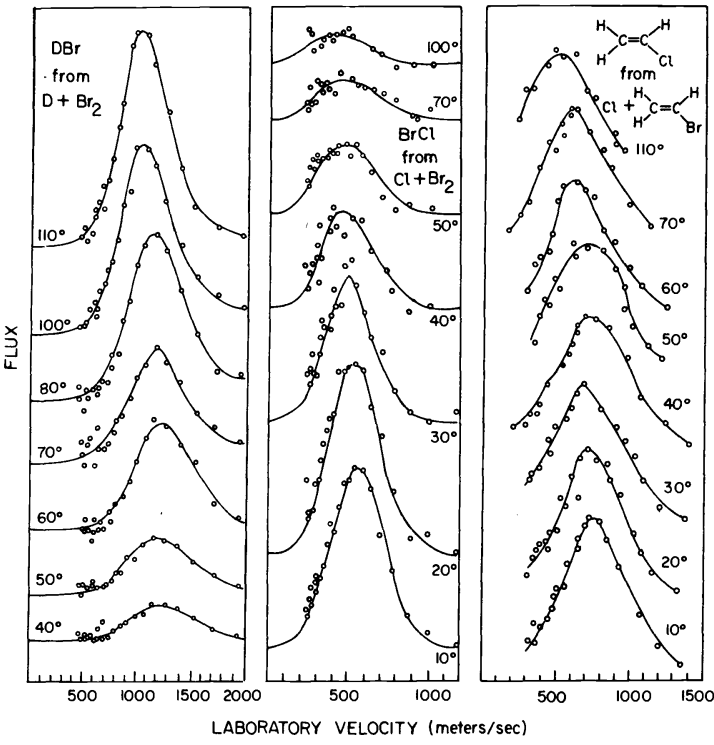

Fig. 1. Velocity distributions of reactive scattering at various angles.

which indicates a collision complex is formed that persists for many vibrational and at least a few rotational periods.

The methods used to generate the reactant beams vary greatly, particularly for reactive atoms or radicals. Beams of alkali atoms ( $\mathrm{Li}, \mathrm{Na}, \mathrm{K}, \mathrm{Rb}, \mathrm{Cs}$ ) or alkaline earth atoms $(\mathrm{Mg}, \mathrm{Ca}, \mathrm{Sr}, \mathrm{Ba})$ are readily obtained by merely heating a suitable oven to moderate temperatures. Beams of hydrogen or deuterium atoms ( $\mathrm{H}$ or $\mathrm{D}$ ) and halogen atoms $(\mathrm{F}, \mathrm{Cl}, \mathrm{Br}, \mathrm{I})$ are produced by thermal dissociation of the parent molecules in high temperature furnaces; for hydrogen, a tungsten furnace at about $3000^{\circ} \mathrm{K}$ is used, for halogens a graphite one at about $1600^{\circ} \mathrm{K}$. Beams of $\mathrm{O}$ and $\mathrm{N}$ atoms are obtained from a radiofrequency discharge source. Beams of $\mathrm{CH}_{3}$ and $\mathrm{CF}_{3}$ radicals are produced by thermal decomposition of suitable parent molecules. Similar sources for $\mathrm{CH}_{2}$ radicals and numerous other species are being developed in current research.

For permanent gases beam production is quite simple. The preferred method ${ }^{11}$ employs a supersonic expansion of the gas from a source chamber at high pressure, often above 100 Torr, through a pin-hole nozzle and into the vacuum. In the nozzle and a short distance beyond (usually less than $1 \mathrm{~cm}$ ), the collision frequency is high and thus the gas flow is hydrodynamic. Further downstream, where the collision frequency becomes very low, the individual molecules move independently and there a true molecular beam is obtained. However, the collisions in the initial hydrodynamic flow bring the beam molecules to a much more uniform direction and velocity than could be obtained from a thermal effusion source. The high source pressure also gives much higher beam intensity than an effusion source. Another important advantage is a very convenient, nonmechanical means of increasing the translational energy of the beam. This is obtained by "seeding" the beam species in a large excess of a lighter nonreactive gas such as helium. The collisions in the nozzle bring the heavy and light molecules to the same velocity, and thus increase the beam translational energy in the ratio of the molecular masses; for example, if molecular chlorine is diluted with helium, this ratio is $\mathbf{1 7}$. The scope of the seeding technique has recently been much extended by a nozzle design suitable for solid or liquid substances. ${ }^{12}$
Selection or analysis of internal states can be accomplished in several ways. For polar molecules, static electric fields are used to deflect or focus the reagent or product and thereby delimit its rotational energy ${ }^{13}$ or orientation. ${ }^{14,15}$ In a few favorable cases, the combined action of static and radiofrequency electric fields has achieved complete resolution of the quantum states of a product molecule, including vibration, rotation, and space quantization. ${ }^{16}$ The most fruitful method for analysis of product energy states is the infrared luminescence technique developed by Polanyi. ${ }^{17}$ This has now provided fully resolved distributions of both vibrational and rotational excitation for $\mathrm{H}+\mathrm{Cl}_{2}, \mathrm{Cl}+\mathrm{HI}, \mathrm{F}+\mathrm{H}_{2}$ and a dozen other reactions. In an important step, McDonald has devised a very sensitive apparatus which extends the luminescence method to large polyatomic product molecules. ${ }^{18}$

Figure 2 illustrates schematically three techniques obtained by coupling lasers with molecular beams. One is photofragment spectroscopy, which deals with unstable electronically excited molecular states. ${ }^{19}$ On absorbing suitable laser light, a molecule in the target beam is abruptly lifted to an excited state in which both its energy and orientation are well-defined. If this state is unstable, the molecule dissociates and launches the fragments on recoil trajectories which give information about the repulsive force and about the shape of the electronic orbital occupied in the excited state.

Another laser technique enables study of the influence of vibrational excitation on reactivity. The original experiment ${ }^{20}$ used the reaction of potassium atoms with hydrogen chloride,

$$
\mathrm{K}+\mathrm{HCl} \rightarrow \mathrm{KCl}+\mathrm{H} .
$$

Like many chemical reactions, this one is energetically inhibited and unexcited $\mathrm{HCl}$ molecules give only a very
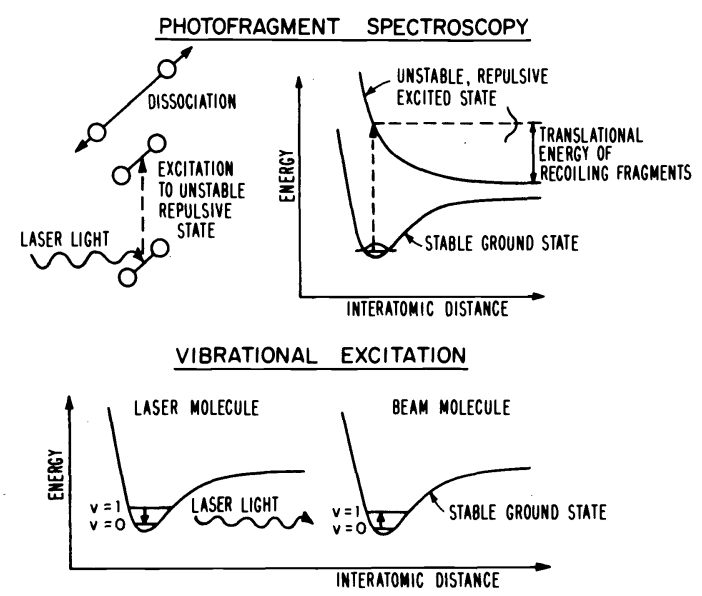

LASER INDUCED FLUORESCENCE

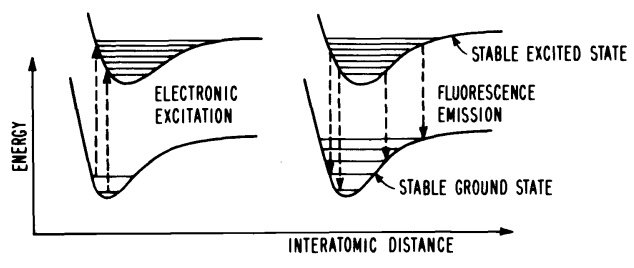

Fig. 2. Experiments employing laser excitation of molecular beams. 
small yield. The experiment exploits a laser in which vibrationally excited $\mathrm{HCl}$ is produced by a "pumping" reaction. This laser emits infrared radiation corresponding to a transition $(v=1 \rightarrow 0)$ between the first excited state and the unexcited ground state of $\mathrm{HCl}$, in various rotational states. The inverse transitions $(v=0 \rightarrow 1)$ are induced when a beam of unexcited $\mathrm{HCl}$ molecules is irradiated by a pulsed beam of the laser light. Detection of the reaction product $\mathrm{KCl}$ is synchronized with the laser pulses. This gives quite directly the ratio of yields for excited $\mathrm{HCl}$ (produced only during the "on" cycle of the pulses) and unexcited $\mathrm{HCl}$ ("off" cycle). The observed ratio is about 100 .

The most recent technique is laser induced fluorescence, which Zare et al. ${ }^{21}$ have shown to be an extremely promising new state-sensitive detector for reactive scattering. In this method, a tunable laser is swept in wavelength. When the laser emission coincides with a molecular absorption line, the product molecule makes a transition to a stable excited electronic state and is detected by observing the subsequent emission from that excited state. The potential sensitivity of the method is extremely high. For a sizeable class of diatomic molecules, it may soon be feasible to measure the angular distribution of the product in an individual rotationvibration state. The method has been applied to the reaction of barium atoms with oxygen molecules,

$$
\mathrm{Ba}+\mathrm{O}_{2} \rightarrow \mathrm{BaO}+\mathrm{O}
$$

and several others. The $\mathrm{BaO}$ appears in a wide range of rotation-vibration states, consistent with a statistical collision complex as indicated by reactive scattering data. As few as $5 \times 10^{4} \mathrm{BaO}$ molecules per cc could be detected in a particular rotation-vibration state. If the $\mathrm{BaO}$ molecules were represented by ping-pong balls numbered to correspond to the quantum states, at this sensitivity the average distance between balls with the same number would be about $2 \mathrm{~km}$.

Both experiment and theory have thus far dealt primarily with energy distributions and angular distributions. However, much more dynamical information can be obtained from study of directional properties related to angular momentum. ${ }^{22}$ For example, for dynamical models which assume abrupt release of a repulsive force as the products separate, ${ }^{7}$ the scattering angle and velocity can be calculated by specifying just the total repulsive energy released, whereas the rotational angular momentum requires in addition the repulsive force as a function of separation distance. The spatial distribution of the product rotational angular momentum is just beginning to be explored in deflection experiments ${ }^{15}$ (by varying the electric field direction) and in trajectory calculations. ${ }^{23}$ For several reactions, the product rotation has been found to be polarized perpendicular to the reactant relative velocity; the polarization is strong in some cases, weak in others. The observable quantities involve averages of the direction cosines relating the product rotational angular momentum to the initial and final relative velocity vectors. There are several such quantities and all vary with the scattering angle and with the relative translational energy and rotation-vibration states of reagents and products. Thus each polarization parameter offers a multidimensional probe of the reaction dynamics analogous to the product angular distribution but with a distinctly different functional dependence.

Figure 3 compares polarization data from a deflection experiment with a model calculation for the $\mathrm{Cs}+\mathrm{CH}_{3} \mathrm{I}$

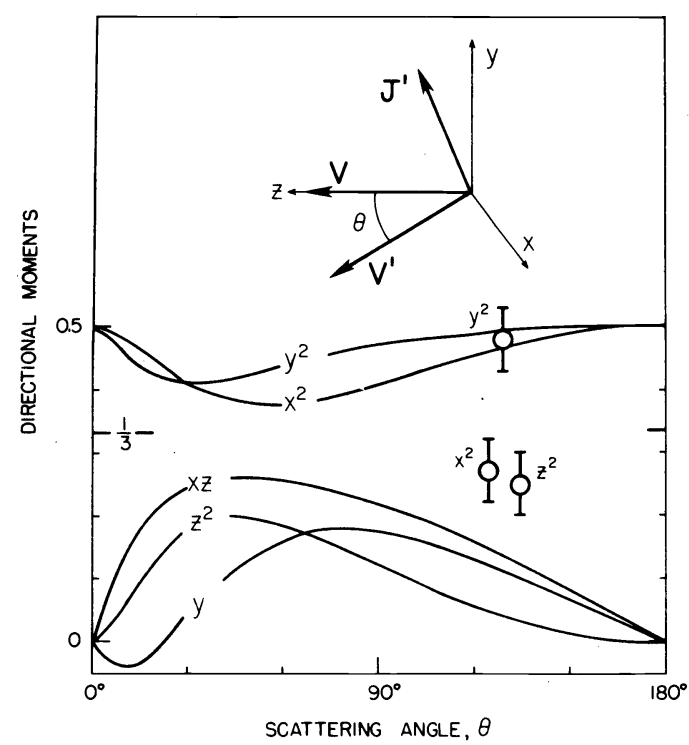

Fig. 3. Moments of direction cosines relating rotational angular momentum $\mathbf{J}^{\prime}$ of $\mathrm{CsI}$ from $\mathrm{Cs}+\mathrm{CH}_{3} \mathrm{I}$ reaction to initial and final relative velocity vectors, $\mathbf{V}$ and $\mathbf{V}^{\prime}$. Curves are calculated from impulsive model, points indicate experimental results. Coordinate system has $z$ axis along $\mathbf{V}$ vector, $y$ axis perpendicular to the scattering plane and $x$ axis in the plane. For an isotropic distribution, the $x^{2}, y^{2}, z^{2}$ moments would be $1 / 3$ and the $x z$ and $y$ moments zero; for a distribution with azimuthal symmetry about $\mathrm{V}$, the $x^{2}$ and $y^{2}$ moments would be equal and the $x z$ and $y$ moments again zero.

reaction..$^{15}$ At present, the experimental method provides only two parameters (a sum rule requires $x^{2}+y^{2}+z^{2}=1$ ), does not determine the angular ảependence, and averages over all energy states. The results are instructive, however. The mean square projection of the CsI rotational momentum perpendicular to the scattering plane is very large. This shows that, as the freshly formed CsI recoils from the collision, its internuclear axis tends to spin in or near the plane of the initial and final relative velocity vectors. Model calculations ${ }^{15}$ and trajectory results $^{23}$ find such alignment is characteristic for $A+$ $B C \rightarrow A B+C$ reactions when strong repulsion occurs between the $B$ and $C$ atoms. Laser-induced fluorescence can be expected to give much more detailed information of this kind since polarization of the product rotation will result in polarization of the fluorescence spectrum. Again, the great sensitivity of the method may allow the polarization of the individual rotation-vibration states to be measured as a function of the scattering angle. Such experiments promise new insight into the "stereochemistry" of reaction dynamics and the underlying potential energy surfaces.

\section{IMPULSIVE REGIME}

For atom exchange reactions a wide domain belongs to direct or impulsive mechanisms. The simplest evidence for this is forward-backward asymmetry of the product angular distribution with respect to the initial relative velocity vector. Such asymmetry implies that the collision complex usually breaks up before it can rotate through a half-turn. Here we consider a favorite example

$$
\mathrm{H}+\mathrm{Cl}_{2} \rightarrow \mathrm{HCl}+\mathrm{Cl} \text {. }
$$

Figure 4 gives a contour map of the distribution in angle 
and velocity of the $\mathrm{HCl}$ product molecules. Successive contours correspond to $10 \%$ increments in intensity. The map is constructed directly from the experimental data (such as Fig. 1), by a geometrical transformation. The coordinate system used has its origin at the center-ofmass of the three atoms, which in this case nearly coincides with the midpoint of the $\mathrm{Cl}_{2}$ bond. In this system, the reagents approach with equal and opposite translational momenta; the direction of the incident $\mathrm{H}$ atoms is designated as $0^{\circ}$ and that of the $\mathrm{Cl}_{2}$ molecule as $180^{\circ}$. The map is symmetric about the $0-180^{\circ}$ line, since the scattering must have azimuthal symmetry about the initial relative velocity. Likewise, in this system, the products recoil with equal and opposite momenta, so the contour map for the $\mathrm{Cl}$ atom (not shown) is the "mirror image" of that for the $\mathrm{HCl}$ molecule.

The product angular distribution is seen to be broad but very anisotropic, with the $\mathrm{HCl}$ recoiling backwards with respect to the incoming $\mathrm{H}$ atom and the $\mathrm{Cl}$ recoiling forwards. The product velocity is very high, about $1600 \mathrm{~m} / \mathrm{sec}$ at the peak of the distribution. About half of the chemical energy released in the bond transformation $(\sim 45 \mathrm{kcal} / \mathrm{mol})$ appears in the translational recoil of $\mathrm{HCl}$ and $\mathrm{Cl}$. The rest appears as vibrational and rotational excitation of the $\mathrm{HCl}$ molecule, which is observed as infrared luminescence. ${ }^{17}$ The form of the angular distribution implies that the preferred configuration of the atoms at the onset of reaction is essentially collinear, $\mathrm{H}-\mathrm{Cl}-\mathrm{Cl}$. The high recoil energy shows that strong repulsive forces are abruptly released as the $\mathrm{Cl}-\mathrm{Cl}$ bond breaks.

This repulsion shows a remarkable resemblance to photodissociation. Figure 4 includes a contour map for the $\mathrm{Cl}$ atoms recoiling from the repulsive electronic state of the $\mathrm{Cl}_{2}$ molecule excited by light absorption (as in Fig. 2, top panel). The direction of the incident photons is $0^{\circ}$. As

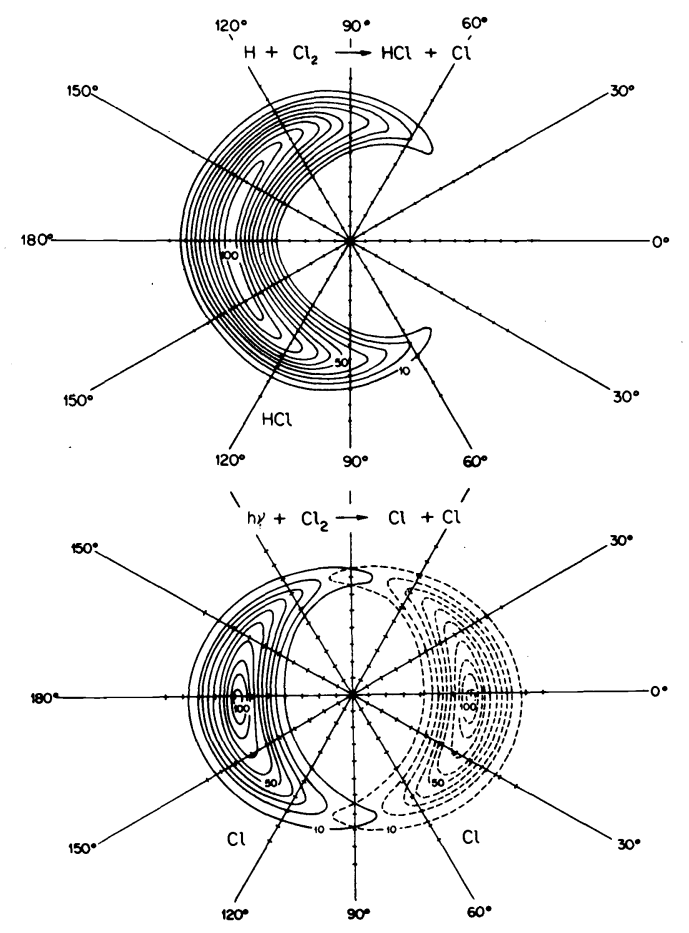

Fig. 4. Comparison of contour maps for $\mathrm{H}+\mathrm{Cl}_{2}$ reactive scattering and for photodissociation of the $\mathrm{Cl}_{2}$ molecule. Tic marks along radial lines indicate velocity intervals of $200 \mathrm{~m} / \mathrm{sec}$. required by the selection rules for absorption, the transition probability is largest for configurations with the $\mathrm{Cl}-\mathrm{Cl}$ axis along the photon direction. The recoil velocity is seen to be nearly the same as in the $\mathrm{H}+\mathrm{Cl}_{2}$ reaction. The large repulsive energy release in photodissociation reflects the strongly antibonding character of the lowestlying unoccupied orbital in the halogen molecule, a $\sigma^{*}$ orbital involving out-of-phase axial overlap of the valence $p$ orbitals of the halogen atoms. A qualitative analysis of the molecular orbitals ${ }^{3}$ for a collinear $\mathrm{H}-\mathrm{Cl}-\mathrm{Cl}$ reaction complex suggests that this antibonding $\sigma^{*}$ orbital also governs the product repulsion.

Other reactions of hydrogen atoms with halogens provide instructive contrasts. As $\mathrm{Cl}_{2} \rightarrow \mathrm{Br}_{2} \rightarrow \mathrm{I}_{2}$, the repulsive energy release becomes a smaller fraction of that in photodissociation and the hydrogen halide angular distribution shifts from backwards to sideways with respect to the $\mathrm{H}$ atom direction. The molecular orbital treatment ${ }^{3}$ relates both trends to the variation in halogen electronegativity, which produces a systematic change in character of the highest occupied orbital in the reaction complex. The $\mathrm{H}+\mathrm{ICl}$ reaction is an especially interesting case. On an energetic purely statistical basis, reaction at the "Cl-end" would be more favorable than at the "I-end", since the $\mathrm{H}-\mathrm{Cl}$ bond strength is much larger than $\mathrm{H}-\mathrm{I}$ (by $32 \mathrm{kcal} / \mathrm{mol}$ ). The molecular orbitals suggest the $\mathrm{H}$ atom should prefer to attack the I-end, however. As a consequence of the electronegativity difference, in $\mathrm{ICl}$ both the highest occupied orbital $\left(\pi^{*}\right)$ and the lowest unoccupied orbital $\left(\sigma^{*}\right)$ are predominantly I atom orbitals. Beam studies ${ }^{10,24}$ show the HI yield is at least comparable to $\mathrm{HCl}$; actually much more $\mathrm{HI}$ is detected but a dispute about experimental factors which discriminate against $\mathrm{HCl}$ remains unresolved. The angular distribution of HI peaks sideways, much like that from the $\mathrm{I}_{2}$ reaction, whereas the $\mathrm{HCl}$ distribution peaks backwards, like that from the $\mathrm{Cl}_{2}$ reaction. An infrared luminescence study ${ }^{25}$ of $\mathrm{H}+\mathrm{ICl}$ observes only $\mathrm{HCl}$ and not $\mathrm{HI}$, but this is compatible with the beam results because the dipole derivative of $\mathrm{HI}$ is exceptionally small and hence the infrared emission is very weak. The $\mathrm{HCl}$ energy distribution has a very unusual bimodal form. Comparison with trajectory calculations ${ }^{25}$ suggests that $18 \%$ of the $\mathrm{HCl}$ results from direct attack at the $\mathrm{Cl}$-end and $82 \%$ from indirect reaction at the I-end. These two reaction modes produce $\mathrm{HCl}$ in low and high rotationvibration states, respectively. At present it is uncertain whether this difference comes from dynamical effects on a single potential surface $e^{25}$ or to reaction on two surfaces. ${ }^{26}$ In any case, the preference for attack at the I-end is consistent with the molecular orbital asymmetry.

Analogous correlations of reaction dynamics with qualitative electronic structure have been pursued for many other atom transfer processes. ${ }^{3,27}$ For example, strong repulsive energy release is often found in reactions of alkali atoms with halomethane molecules. The low alkali ionization potential allows the valence electron to transfer to the target molecule. In many cases the electron enters a strongly antibonding orbital with a radial node between the central carbon atom and an adjacent halogen atom, which is then ejected as a halide anion and combines with the alkali cation to form the product salt molecule. The dissociative electron attachment suffered by the reagent molecule resembles photodissociation. Examples involving orbital asymmetry are numerous. Thus, reactions of $\mathrm{Br}, \mathrm{O}$, and $\mathrm{CH}_{3}$ with ICI all form predominantly iodides. Analysis of triatomic molecular 
orbitals ${ }^{28}$ has led to a general criterion which we refer to as "the electronegativity ordering rule." This predicts that $\mathrm{H}-X-Y$ will be a more favorable conformation than $\mathrm{H}-$ $Y-X$, if $X$ is less electronegative than $Y$ (where these are any atoms with $p$ electrons.) Likewise, in $X-Y-Z$ systems, the preferred conformation has the least electronegative atom in the middle. If the electronegativity difference becomes so large that electron transfer occurs, other considerations enter. ${ }^{29}$ However, otherwise this rule holds for almost all known stable triatomic molecules, linear or bent, and it now appears to apply also to preferred reactive conformations.

\section{PERSISTENT COMPLEX REGIME}

When the duration of a reactive collision is long compared to vibrational periods of the transient collision complex, product formation is indirect since the interacting atoms or bonds undergo multiple encounters during the collision. The distributions in energy and angle of the scattered products give information about the unimolecular decomposition of the lingering or persistent complex. The beam technique thus offers a single-collision version of the chemical activation method of unimolecular kinetics. ${ }^{30}$ This is illustrated here for the reaction of chlorine atoms with vinyl bromide, ${ }^{31}$

$$
\mathrm{Cl}+\mathrm{CH}_{2}=\mathrm{CHBr} \rightarrow \mathrm{CH}_{2}=\mathrm{CHCl}+\mathrm{Br}
$$

Figure 5 gives the contour map. The symmetrical forward-backward peaking with respect to the initial relative velocity shows that the intermediate collision complex persists long enough to rotate several times and thereby "forget" the approach direction before decomposing to form the products. This implies that the mean lifetime of the complex exceeds about $5 \times 10^{-12} \mathrm{sec}$. That is much longer than the periods of typical vibrational motions within the complex, which are about $10^{-13} \mathrm{sec}$, and much longer than the collision duration for impulsive reactions, which is also typically of the order of $10^{-13}$ or shorter. The relatively long lifetime of the complex
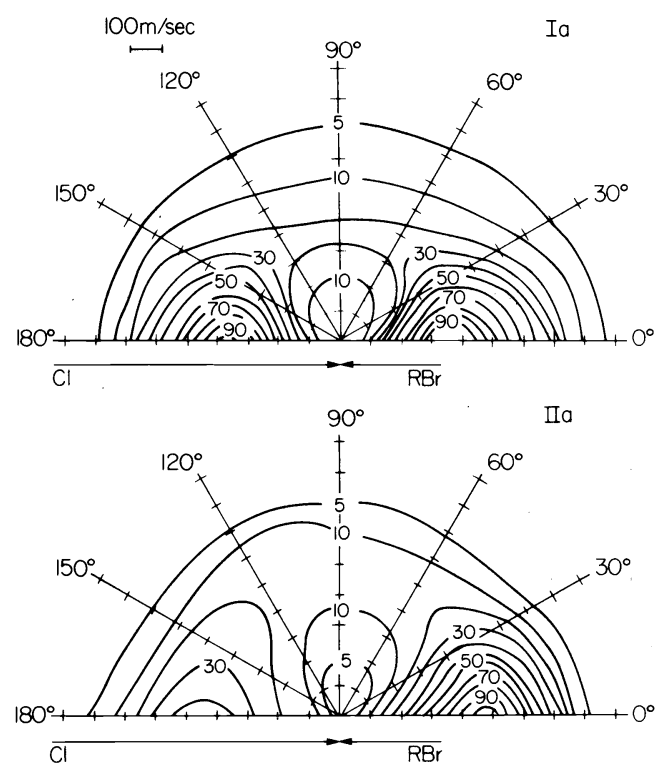

Fig. 5. Contour maps for bromo-olefin products from reactions of chlorine atoms with (Ia) vinyl bromide and with (IIa) allyl bromide. Tic marks along radial lines indicate velocity intervals of $100 \mathrm{~m} / \mathrm{sec}$. requires that attractive rather than repulsive forces govern the reaction. In this example, the complex indeed corresponds to a known, stable species, the chlorobromoethyl radical,<smiles>ClCCl</smiles>

The intermediate radical is vibrationally excited (by $\sim 30 \mathrm{kcal} / \mathrm{mol}$ ) since energy is generated by addition of the chlorine atom, which converts the cargon-carbon double bond to a single bond and forms a new carbon-chlorine bond. In an ordinary chemical environment, the vibrational excitation would be quickly degraded by subsequent collisions but this does not occur in a beam experiment. The vibrational excitation thus remains "trapped" in the complex, shuffling among the various bonds until eventually one bond ruptures and the complex dissociates. For this system, the only dissociation paths energetically accessible involve reforming the double bond and releasing either the $\mathrm{Cl}$ atom or the $\mathrm{Br}$ atom. The latter path is much more favorable since the $\mathrm{C}-\mathrm{Br}$ bond is weaker than the $\mathrm{C}-\mathrm{Cl}$ bond. The long lifetime of the complex allows the vibrational excitation energy to become random before decomposition. Thus, the product energy distribution can be predicted from a simple statistical model, akin to the Rice-RamsbergerKassel-Marcus theory for unimolecular reactions. ${ }^{32}$

The main qualitative features of the statistical theory ${ }^{33}$ are illustrated in Fig. 6. Consider first a complex which is not rotating. In partitioning the available energy among relative translation, vibration, and rotation of the products, the statistically favored situation puts only a small part of the energy into translation, since the vibrational and rotational modes are more numerous. Thus, the probability distribution declines rather rapidly with increase in product translational energy. This decline becomes more rapid as the number of atoms in the complex increases and hence the number of vibrational modes increases. Another important effect enters when the complex is rotating, however. The energy in centrifugal motion of the rotation complex is not available for statistical distribution among the other modes. On decomposition of the complex this centrifugal energy is converted into relative motion of the emerging product molecules. This changes the shape of the product translational energy distribution. The region of low translational energy is determined by the centrifugal contribution, the region of high translational energy by the statistical contribution.

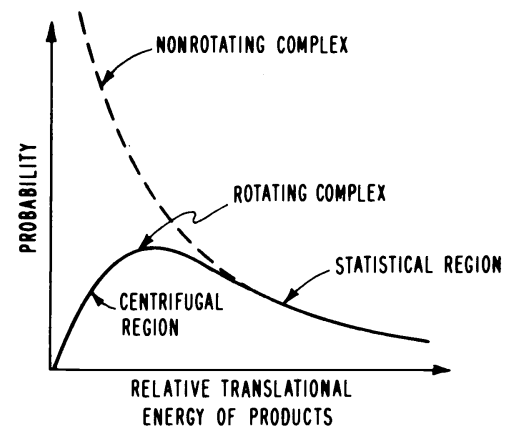

Fig. 6. Distribution of product relative translational energy predicted by statistical theory. 
The shape of the product angular distribution is also related to rotational properties of the complex. ${ }^{34}$ How this comes about may be illustrated by two limiting cases shown in Fig. 7. Consider first a complex having all atoms collinear in the critical configuration or transition-state for decomposition. The rotation of such a complex occurs about an axis perpendicular to the line of atoms. If the complex rotates a few times before dissociating, the products are spread uniformly over all azimuthal angles about the rotation axis, like water from a lawn sprinkler. The total distribution is obtained by averaging uniformly over all orientations of the sprinkler with respect to the direction of approach of the reactants. The result corresponds to the intersections of circles of latitude and longitude on a globe. The product intensity becomes very high in the "polar" regions, which represent scattering directions parallel or nearly parallel to the initial reactant approach, but the intensity is low in the "equatorial" regions.

Consider next a complex having all atoms situated in two coaxial hoops which maintain a face-to-face configuration in the transition-state. Suppose also that the rotational motion of the complex consists entirely of the spinning of these hoops about their axis of separation as the complex dissociates. Since the axis of rotation of the complex is again perpendicular to the direction of reactant approach, in this case the product separation axis must be perpendicular to it also. The product intensity therefore is high in the equatorial regions and low in the polar regions.

The arrangement of atoms and the rotational motions in the transition-state thus can leave its imprint in the product angular distribution, even though the complex often dissociates after a few rotations. A complex such as the chlorobromoethyl radical has some atoms situated close to or on the axis of product separation and others located well away from that axis. The rotation of the complex hence corresponds to a superposition of the limiting cases of Fig. 7. There is both centrifugal rotation of the separation axis

\section{COLLINEAR COMPLEX}
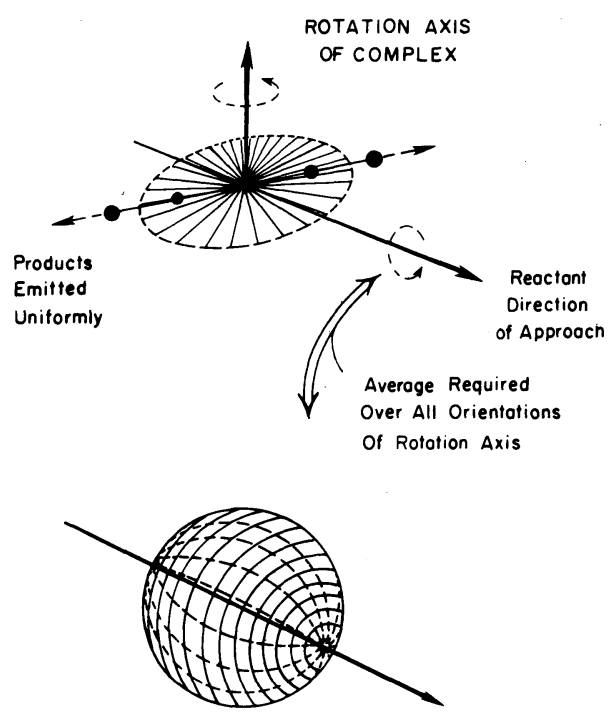

PROOUCT DISTRIBUTION FAVORS POLAR REGIONS and spinning of the product molecules about that axis. The shape of the angular distribution reveals the relative contribution of the centrifugal and spinning motions. This can be regarded as roughly analogous to the rotational spectrum of a stable molecule, for which the moments of inertia about the principal axes indicate the mass-weighted mean square average distances of atoms from those axes. In the chlorobromoethyl case, the pronounced forwardbackward peaking indicates that centrifugal momentum is dominant in the dissociation of the collision complex.

Figure 8 compares the observed and calculated product translational energy distributions for three reactions: oxygen atoms and bromine ${ }^{35}$ chlorine atoms with vinyl bromide, ${ }^{31}$ and cesium with sulfur hexafluoride. ${ }^{36}$ The centrifugal contribution and the number of contributing vibrational modes vary considerably among these reactions but the statistical theory gives good agreement in each case. Long-range attraction of the form $-C / r^{6}$ was assumed for both the reactants and products in evaluating the centrifugal factor; the force constants $C$ were estimated from customary approximations involving molecular polarizabilities. All vibrational modes were assumed to be active in intramolecular energy transfer. For the vinyl bromide reaction, however, the contributions from hydrogen atom motions are almost negligible because of the large mean vibrational spacings for these modes. ${ }^{31}$ This simple approximate theory has given good agreement with translational energy data for many other reactions. ${ }^{3}$ Similar results have been obtained with the corresponding angular distribution theory. Thus, in the persistent complex regime the transition-state approximation provides a useful interpretative tool.

Figure 5 includes a contour map for the reaction of chlorine atoms with allyl bromide,

$$
\mathrm{Cl}+\mathrm{CH}_{2}=\mathrm{CHCH}_{2} \mathrm{Br} \rightarrow \mathrm{ClCH}_{2} \mathrm{CH}=\mathrm{CH}_{2}+\mathrm{Br} \text {. }
$$

The forward peak of the angular distribution is more
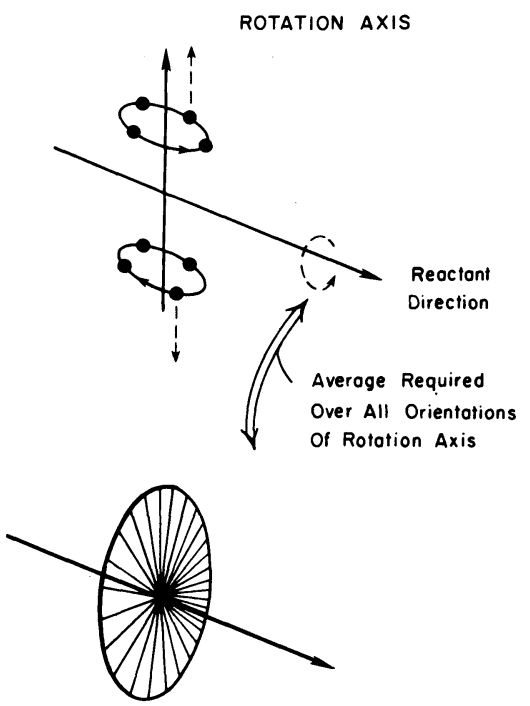

PROOUCTS CONFINED

TO EQUATORIAL REGION

Fig. 7. Angular distribution of products predicted by statistical theory for two special cases. 


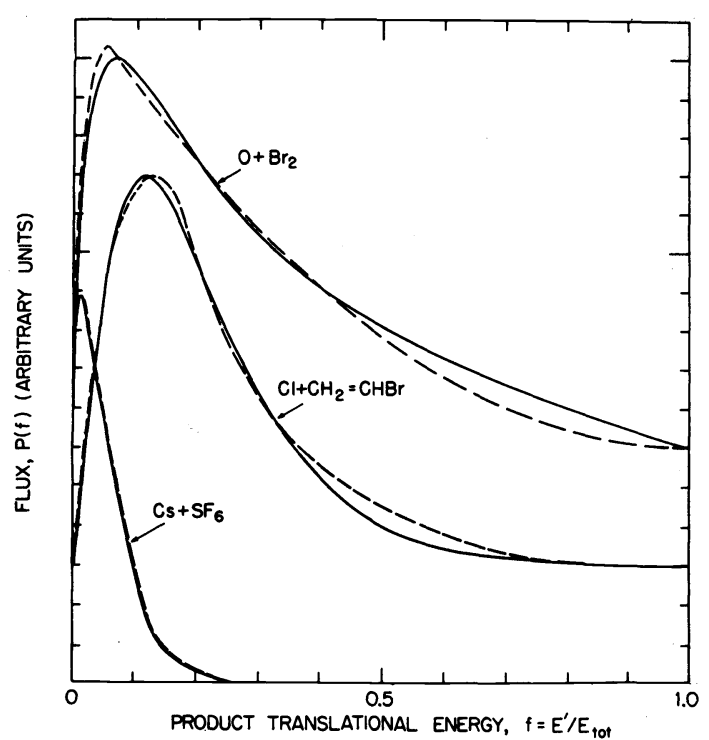

Fig. 8. Comparison of product relative translational energy distributions derived from experiment (full curves) and from statistical theory (dashed curves).

pronounced than the backward peak, by a factor of about three. This indicates that the complex is dissociating as it rotates. For such cases an "osculating complex" model ${ }^{37}$ has been formulated in which the dissociation occurs with an exponential distribution of lifetimes. Comparison with the data shows the mean lifetime of the complex is about one-half of a rotational period, or about $1 \times 10^{-12} \mathrm{sec}$. In accord with this shorter lifetime, the product translational energy distribution for this reaction is markedly nonstatistical. It is displaced upwards, indicating the only about two-thirds of the vibrational modes associated with the five heavy atoms (carbon or halogen atoms) are active in the intramolecular energy transfer before dissociation of the complex.

In the context of other work, the contrast between the reactive scattering of chlorine atoms with vinyl bromide and allyl bromide suggests a qualitative difference in mechanism. The vibrational excitation of the intermediate chlorobromoalkyl radical is practically the same $(\sim 30 \mathrm{kcal} / \mathrm{mol})$ and the dissociation energy for bromine atom emission from these radicals derived from thermochemical data is also about the same ( $\sim 18-$ $20 \mathrm{kcal} / \mathrm{mol}$ ). The customary theory of unimolecular processes predicts that the allylic reaction should proceed more slowly and hence show more nearly statistical behavior, since it involves one more heavy atom and therefore three more accessible vibrational modes than the vinylic reaction. The lifetime for bromine atom emission estimated from the theory is less than about $2 \times 10^{-12} \mathrm{sec}$ for both the vinylic and allylic systems. Some special feature apparently intervenes to make the vinylic reaction more statistical than the allylic reaction. The initial stage in both reactions almost certainly involves attack on the double bond rather than the $\mathrm{C}-\mathrm{Br}$ bond $\mathrm{d}^{38}$ and there is extensive evidence for anti-Markovinkov addition to olefins. ${ }^{39}$ Thus, a likely hypothesis ${ }^{31}$ is that the vinylic reaction proceeds via a 1,2-chlorine atom migration, whereas the allylic reaction goes via a 1,3-bond migration,

$\mathrm{Cl}+$<smiles>C=CCBr</smiles><smiles>ClCCCBr</smiles>

Since the heavy atom migration should be much slower than the bond migration, this might provide a rate-limiting process which makes the vinylic reaction more statistical than the allylic one.

These mechanisms require the product chlorolefin to have $\mathrm{Cl}$ on the carbon to which $\mathrm{Br}$ was originally bonded in the vinylic case, but on the "most distant" carbon in the allylic case. This was verified by analysis of the fragment ion mass spectra of chlorolefins formed in corresponding reactions with a methyl group added to "label" one or another carbon atom. ${ }^{31}$ The vinylic reactants,<smiles>C/C=C\Br</smiles>

were indeed found to yield only the chlorolefin with $\mathrm{Cl}$ at the original $\mathrm{Br}$ position in each case. For the allylic reactants,<smiles>CC=CCBr</smiles>

and<smiles>C=CC(C)Cl</smiles><smiles>C=CC(C)Br</smiles>

The methyl substitutions also produce interesting changes in the reactive scattering. For the vinylic cases, the angular distributions show variations which reflect changes in the rotational motions caused by the methyl group. For the allylic cases, the product translational energy distributions become statistical, as if the methyl group diverts the intramolecular energy flow and thereby makes it more nearly random.

Similar studies have now been conducted for about thirty other families of reactions governed by persistent collision complexes. ${ }^{3,4}$ In almost every case the intermediate complex corresponds to a known chemical species or to an electronic structure with favorable bonding overlaps. As with the chlorobromolefins considered here, however, many such systems show nonstatistical features. We cite three examples. (1) Analogous reactions of fluorine atoms with olefins have been extensively studied by Lee and coworkers in crossedbeams $^{40}$ and by McDonald in i.r. luminescence. ${ }^{18}$ The vibrational excitation of the intermediate radicals is quite high $(\sim 60 \mathrm{kcal} / \mathrm{mol})$ by virtue of the large C-F bond strength, and several competing dissociation channels involving scission of $\mathrm{C}-\mathrm{C}$ bonds and migration of $\mathrm{CH}$ groups or $\mathrm{H}$ atoms become accessible. The translational and vibrational energy was found to show systematic deviations from statistical behavior; often the population of high modes is low and that of low modes is high. (2) Reactions of alkali atoms with alkali halide molecules
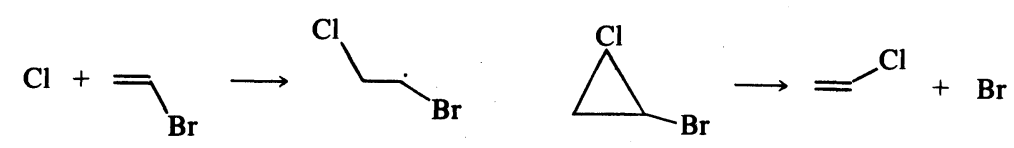
such as

$$
\mathrm{Na}+\mathrm{K}^{+} \mathrm{Cl}^{-} \rightleftarrows(\mathrm{NaK})^{+} \mathrm{Cl}^{-} \rightarrow \mathrm{Na}^{+} \mathrm{Cl}^{-}+\mathrm{K}
$$

have also been much studied. ${ }^{34,41}$ The reactant and product salt molecules are essentially ion-pairs and electronic structure calculations ${ }^{42}$ show the ionic dialkali halide $(\mathrm{NaK})^{+} \mathrm{Cl}^{-}$is likewise a stable species. Statistical models give good agreement with the product translational and angular distributions but for more than 20 cases overestimate the ratio of reactive to nonreactive decay of the collision complex, often by a factor of 3-5 or more. This has been attributed to preferred reaction geometry. ${ }^{34}$ The potential surfaces predict the most stable configuration of the dialkali halide complex is triangular but the most favorable direction of approach is collinear, with $\mathrm{Na}$ attacking the $\mathrm{K}^{+}$end of the salt molecule. Since the centrifugal momentum in the collision often restrains the roughly collinear $(\mathrm{NaK})^{+} \mathrm{Cl}^{-}$configurations from bending into the triangular configurations required for reaction, this "wrong-end" approach strongly favors nonreactive decay of the complex. (3) Reactions of oxygen atoms with halogen molecules ${ }^{35}$ also conform to the statistical complex model yet show clear evidence for preferred reaction geometry. In particular, the $\mathrm{O}+\mathrm{ICl}$ reaction yields only $\mathrm{IO}+\mathrm{Cl}$ and not $\mathrm{ClO}+\mathrm{I}$, again the result consistent with the "electronegativity ordering rule".

\section{FACILE MOLECULAR REACTIONS}

One of the chief criteria invoked in postulating elementary steps in chemical reaction mechanisms is that only processes involving attack by an open-shell atom or free radical have very low activation energies, less than 5 or $10 \mathrm{kcal} / \mathrm{mol}$. Reactions of molecules with molecules, which involve the concerted making and breaking of two or three pairs of bonds, typically have activation energies above $20 \mathrm{kcal} / \mathrm{mol}$ when fully "allowed" in the Woodward-Hoffmann sense and about $40 \mathrm{kcal} / \mathrm{mol}$ when "forbidden". It is thus instructive that recent molecular beam experiments find certain classes of bimolecular and termolecular reactions of diatomic molecules proceed with remarkably low activation energies, zero or at most a few $\mathrm{kcal} / \mathrm{mol}$.

Before describing these results, we briefly consider related work on the isotopic exchange of hydrogen and deuterium,

$$
\mathrm{H}_{2}+\mathrm{D}_{2} \rightarrow 2 \mathrm{HD} \text {. }
$$

This is a prototypical example (although numerically atypical) of a "forbidden" four-center reaction. It has aroused much interest because a severe conflict between theory and experiment has persisted for several years. Figure 9 illustrates the correlation of reactant and product molecular orbitals for the simplest case of a coplanar path proceeding through a square transition state. ${ }^{43}$ The nodal properties of the composite orbitals formed by in-phase and out-of-phase (dark shading) overlap of the diatomic bonding $\sigma$ and antibonding $\sigma^{*}$ orbitals require the four-center reaction to raise two of the four electrons from $\sigma$ to $\sigma^{*}$ orbitals. This suggests the potential energy barrier should be comparable to or larger than the $\mathrm{H}_{2}$ dissocation energy (about $110 \mathrm{kcal} / \mathrm{mol}$ ). Electronic structure calculations ${ }^{44}$ indeed find the square planar $\mathrm{H}_{4}$ transition state lies more than $140 \mathrm{kcal} / \mathrm{mol}$ above the $\mathrm{H}_{2}+\mathrm{H}_{2}$ ground state. A variety of other possible reaction paths have also been examined, including tetrahedral,
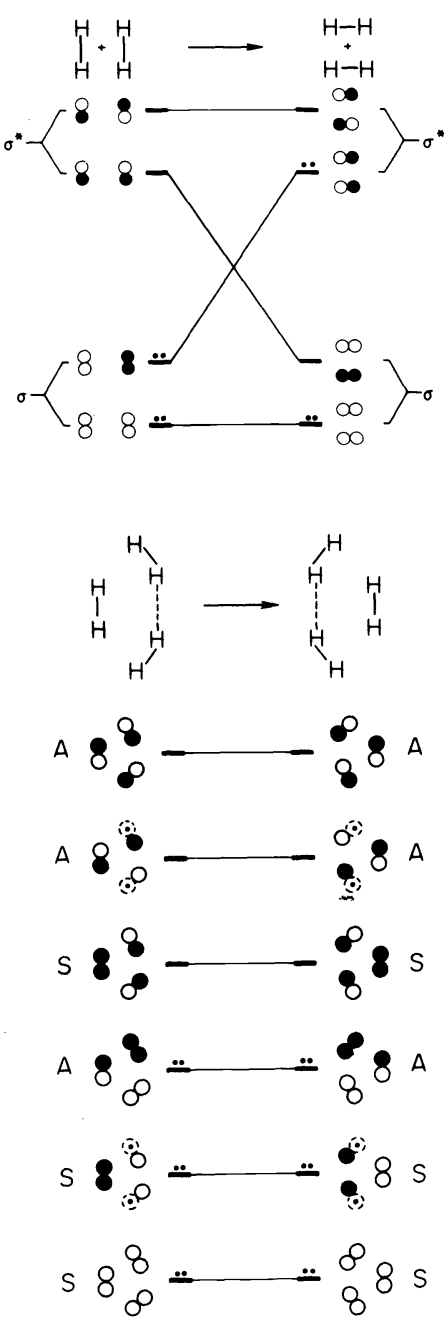

Fig. 9. Orbital correlation diagrams for $\mathrm{H}_{4}$ and $\mathrm{H}_{6}$ systems.

trapezoidal, rhomboid, kite, and Y-or T-shaped transition states; all appear tolie above the $\mathrm{H}_{2}$ dissociation energy.

Experiments on hydrogen-deuterium exchange in shock tubes ${ }^{45}$ find an activation energy of only about $40 \mathrm{kcal} / \mathrm{mol}$. Elegant evidence that vibrationally excited $\mathrm{H}_{2}$ or $\mathrm{D}_{2}$ are involved has been obtained by use of a stimulated Raman laser. ${ }^{46}$ The observed rate law is complex, as a consequence of multistage vibrational energy transfer, and the empirical activation energy is not simply related to the potential energy barrier. However, the HD yields are roughly $6-9$ orders of magnitude too large to reconcile with the $\mathrm{H}_{4}$ potential surface calculations.

Figure 9 includes a correlation diagram for the termolecular, six-center reaction proceeding through an $\mathrm{H}_{6}$ hexagon. This is a fully "allowed" process. For simplicity, the path shown preserves a symmetry plane bisecting one of the initial and final $\mathrm{H}_{2}$ bonds and the orbitals are classified as symmetric (S) or antisymmetric (A) with respect to that plane, but the nodal properties which govern the qualitative correlations are similar for other paths. Wright ${ }^{47}$ proposed that the hydrogendeuterium exchange might go by this six-center route and showed the energy barrier for an $\mathrm{H}_{6}$ hexagon is at least lower than for an $\mathrm{H}_{4}$ square. Following the recent beam results on termolecular reactions, Dixon ${ }^{48}$ undertook 
more accurate calculations for $\mathrm{H}_{6}$ and found the potential barrier in fact lies far below the $\mathrm{H}_{2}$ dissociation energy. Figure 10 shows some of his results obtained with successively better wavefunctions. By extrapolation, the potential barrier for the $\mathrm{H}_{6}$ hexagon is estimated to be no higher than $60 \mathrm{kcal} / \mathrm{mol}$ and perhaps substantially less. Kinetic calculations ${ }^{48}$ show that, despite the reduced probability of termolecular collisions as compared with bimolecular ones, the six-center processes

$$
\begin{aligned}
& \mathrm{H}_{2}+\mathrm{H}_{2}+\mathrm{D}_{2} \rightarrow 2 \mathrm{HD}+\mathrm{H}_{2} \\
& \mathrm{H}_{2}+\mathrm{D}_{2}+\mathrm{D}_{2} \rightarrow 2 \mathrm{HD}+\mathrm{D}_{2}
\end{aligned}
$$

with vibrationally excited reactants are consistent with the experimental data. A special form of "vibrational catalysis" becomes possible here. If a substantial part of the energy needed to surmount the potential barrier goes into vibration of the $\mathrm{H}_{2}$ or $\mathrm{D}_{2}$ regenerated by the six-center reaction, rapid vibration-vibration energy transfer in bimolecular collisions of these molecules with the ambient gas can raise the vibrational temperature significantly and hence accelerate the overall exchange rate. It may be possible to test the termolecular mechanism by looking for this effect in future experiments.

The crossed beam studies of molecular reactions were prompted by the classic work of Sullivan ${ }^{49}$ on the favorite "textbook" case,

$$
\mathrm{H}_{2}+\mathrm{I}_{2} \rightarrow 2 \mathrm{HI} \text {. }
$$

He showed this does not occur as a four-center reaction but involves dissociation or near-dissociation of $I_{2}$ followed by $\mathrm{I}+\mathrm{H}_{2}+\mathrm{I}$. Noyes ${ }^{50}$ likewise cast doubt on $\mathrm{Br}_{2}+\mathrm{I}_{2}$ and other reactions long presumed to be bimolecular. The subsequent orbital correlation arguments ${ }^{43}$ indicated that most bimolecular exchange reactions of covalently-bonded diatomic molecules are forbidden. In this context, several beam experiments were tried in pursuit of four center reactions. For example, the $\mathrm{HI}+\mathrm{DI}$ reaction was examined ${ }^{51}$ using the seeded supersonic beam technique and no detectable HD yield found at collision energies far above the empirical activation energy. This is certainly an allowed reaction, at

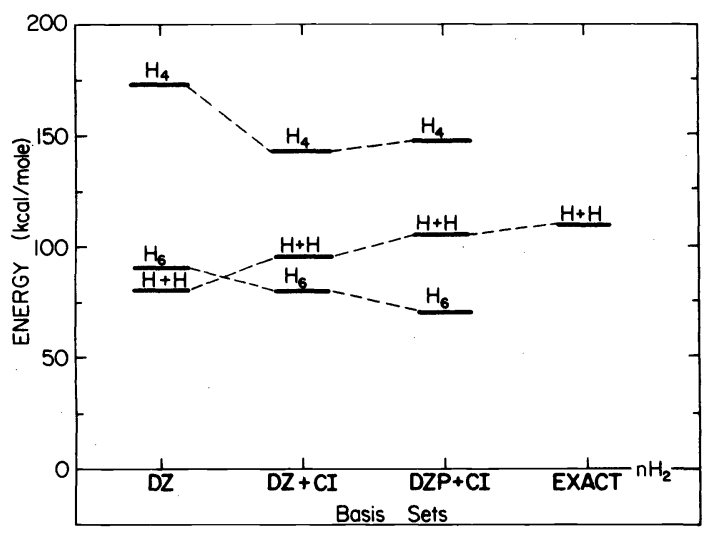

Fig. 10. Energy of square planar $\mathrm{H}_{4}$ relative to $2 \mathrm{H}_{2}$ and hexagonal planar $\mathrm{H}_{6}$ relative to $3 \mathrm{H}_{2}$, for various approximate electronic structure calculations: $\mathrm{DZ}$ denotes double zeta; $\mathrm{CI}$ configuration interaction with all single and double excitations; $\mathrm{P}$ polarization. Corresponding results for the dissociation energy of $\mathrm{H}_{2}$ are also shown. least as the reverse of $\mathrm{I}+\mathrm{HD}+\mathrm{I}$, but apparently vibrational rather than translational activation is required. However, a sizeable class of four-center reactions was found $^{52}$ which proceeds readily at thermal collision energies of a few $\mathrm{kcal} / \mathrm{mol}$. These all involve ionic bonds.

The simplest case is the exchange reaction of two alkali halides such as

$$
\mathrm{Cs}^{+} \mathrm{Cl}^{-}+\mathrm{K}^{+} \mathrm{I}^{-} \rightarrow \mathrm{Cs}^{+} \mathrm{I}^{-}+\mathrm{K}^{+} \mathrm{Cl}^{-} .
$$

This might be termed a "no-electron" reaction, since the salt molecules are essentially closed-shell ion-pairs. Accordingly, no restraints are imposed by orbital correlations. Since alkali halides form rhombic quadrupolar dimers with dissociation energy $\sim 30-50 \mathrm{kcal} / \mathrm{mol}$, the potential surface for the exchange reaction has a deep basin. The beam data are indeed consistent with this, except that the ratio of nonreactive to reactive decay of the complex was 2 or 3 times larger than statistical. ${ }^{34}$ As in the analogous atom + salt case, this can be attributed to geometrical isomerism. Ionic model calculations predict less stable, linear chain isomers exist in addition to the rhombic dimer. These linear chain isomers may often dissociate nonreactively rather than rearrange to the cyclic form required for the exchange process; again, this is especially likely when the centrifugal angular momentum in the collision keeps the chain "ends" apart. Trajectory calculations ${ }^{53}$ for this reaction have confirmed the role of geometrical isomerism.

Four-center reactions involving one ionic and one covalent bond ${ }^{52}$ also proved to be facile at thermal collision energies; for example,

$$
\mathrm{Cs}^{+} \mathrm{Br}^{-}+\mathrm{ICI} \rightarrow \mathrm{Cs}^{+} \mathrm{Cl}^{-}+\mathrm{IBr} .
$$

The collision complex very likely corresponds to a trihalide salt, $\mathrm{Cs}^{+}(\mathrm{BrICl})^{-}$. Although apparently unknown in the gas phase, trihalides have been much studied in solution and in the solid state. In agreement with molecular orbital theory, ${ }^{28}$ the trihalide anions are linear or nearly linear, the middle atom is always the least electronegative (I, in this case) and it has a small positive charge whereas the end atoms share the negative charge. Striking evidence for this structure appears in some features of the reactive scattering. There is no observable yield of $\mathrm{Cs}^{+} \mathrm{I}^{-}+\mathrm{BrCl}$, even at collision energies more than $20 \mathrm{kcal} / \mathrm{mol}$ above the energetic threshold for this channel. Furthermore, as seen in Fig. 11, the product angle-velocity contour map has a very unusual skewed shape. The lefthand product peak has distinctly higher intensity and velocity than the righthand peak. This shows

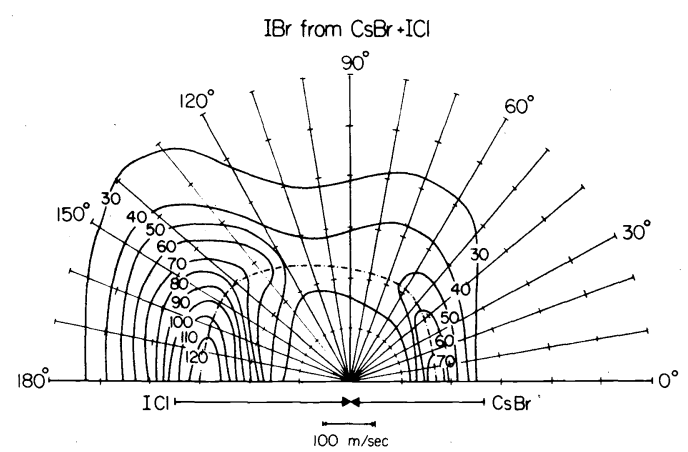

Fig. 11. Contour map of $\mathrm{IBr}$ product distribution from $\mathrm{CsBr}+\mathrm{ICl}$ reaction at collision energy of $3.9 \mathrm{kcal} / \mathrm{mol}$. 
that collisions from which $\mathrm{IBr}$ and $\mathrm{CsCl}$ rebound backwards with respect to the incident $\mathrm{ICl}$ and $\mathrm{CsBr}$, respectively, are more probable and involve more repulsive energy release than collisions from which $\mathrm{IBr}$ and $\mathrm{CsCl}$ emerge in the same direction as the incident $\mathrm{ICl}$ and $\mathrm{CsBr}$, respectively. These properties are consistent with the expectation that in $\mathrm{Cs}^{+} \mathrm{Br}^{-}+\mathrm{ICl}$ reactive configurations, $\mathrm{Br}^{-}$tends to be collinear with $\mathrm{ICl}$ while $\mathrm{Cs}^{+}$in "reaching" for $\mathrm{Cl}$ must come near the central I atom. The positive charge acquired by I as the trihalide forms then repels $\mathrm{Cs}^{+}$, which picks up the emerging $\mathrm{Cl}^{-}$ and departs quickly in the direction opposite to the incident salt.

Similar four-center ionic + covalent reactions such as $\mathrm{CsF}+\mathrm{HCl}$ were likewise found to go with near zero activation energy. ${ }^{52}$ It will be interesting to see what happens for systems with only partial ionic bonds. A large class of four-center 1,2 elimination reactions of haloolefins which have been described in terms of a semi-ion pair transition state ${ }^{54}$ have activation energies near $20-30 \mathrm{kcal} / \mathrm{mol}$, again much lower than comparable covalent + covalent cases.

Six-center reactions involving three covalent diatomic molecules were found in beam experiments ${ }^{55}$ undertaken in response to tantalizing evidence obtained by Noyes ${ }^{50}$ for a third order reaction in solution,

$$
\mathrm{Br}_{2}+2 \mathrm{I}_{2} \rightarrow 2 \mathrm{IBr}+\mathrm{I}_{2}
$$

For convenience we examined the analogous system involving a bromine molecule and two chlorine molecules. In preliminary work, King and Dixon established that under single collision conditions the four-center process

$$
\mathrm{Br}_{2}+\mathrm{Cl}_{2} \rightarrow 2 \mathrm{BrCl}
$$

yields no product attributable to bimolecular reaction, at translational energies up to $\sim 25 \mathrm{kcal} / \mathrm{mol}$. The prospect for observing the termolecular $\mathrm{Br}_{2}+2 \mathrm{Cl}_{2}$ process appeared dubious. Analogy to other "allowed" six-center cases (such as Diels-Alder reactions) suggested that the potential barrier might well be $\sim 20 \mathrm{kcal} / \mathrm{mol}$, and it might also require chiefly vibrational rather than translational excitation. Thus, there was consternation when King and Dixon found large yields of $\mathrm{BrCl}$ which appeared to come from the termolecular process even at thermal collision energies of only $\sim 3 \mathrm{kcal} / \mathrm{mol}$. Their results have now withstood numerous refinements in technique and consistency tests, however, and similar results have been obtained for several other systems involving concerted transformation of three pairs of bonds with practically no activation energy. .8 $^{4}$

The beam technique for study of termolecular reactions again exploits supersonic nozzles. Expansion of chlorine gas through a pin-hole generates dimeric $\mathrm{Cl}_{2} \cdots \mathrm{Cl}_{2}$ molecules, held together by a weak van der Waals bond ${ }^{56}$ with dissociation energy of $1-2 \mathrm{kcal} / \mathrm{mol}$ and equilibrium bond distance roughly $4.3 \AA$ (between centroids of the monomers). Source conditions were eventually found for which the dimer fraction is a few percent but trimers and higher polymers are almost undetectable. This has allowed study of three termolecular reaction paths,

$$
\begin{aligned}
\mathrm{Br}_{2}+\mathrm{Cl}_{2} \cdots \mathrm{Cl}_{2} & \rightarrow 2 \mathrm{BrCl}+\mathrm{Cl}_{2} \\
& \rightarrow \mathrm{BrCl} \cdots \mathrm{Cl}_{2}+\mathrm{BrCl} \\
& \rightarrow \mathrm{Br}_{2} \cdots \mathrm{Cl}_{2}+\mathrm{Cl}_{2} .
\end{aligned}
$$

As usual, the reactant beams were monitored and the products detected with mass and time-of-flight velocity analysis. The $\mathrm{BrCl}, \mathrm{BrCl}_{3}$, and $\mathrm{Br}_{2} \mathrm{Cl}_{2}$ signals all correlated with the $\left(\mathrm{Cl}_{2}\right)_{2}$ signal. Marked differences were found in the dependence on collision energy and the product angle-velocity distributions which show the signals come from three distinct reaction modes; in particular, very little of the $\mathrm{BrCl}$ signal can be attributed to fragmentation of $\mathrm{Br}_{2} \mathrm{Cl}_{2}$ in the detector. Inelastic scattering of $\left(\mathrm{Cl}_{2}\right)_{2}$ with velocity changes due to translation-to-vibration energy transfer is also very prominent. At higher energies collision induced dissociation to form $\mathrm{Br}_{2}+\mathrm{Cl}_{2}+\mathrm{Cl}_{2}$ becomes the major process.

Figure 12 compares the reaction yields and the dependence on collision energy. ${ }^{55}$ Since (R1) and (R2) involve making and breaking three pairs of chemical bonds, these paths presumably require the incident bromine molecule to interact with both chlorine molecules in a cyclic configuration. However, (R3) merely exchanges van der Waals bonds among the three molecules without disrupting the chemical bonds. Path (R3) thus is accessible for many noncyclic collisional orientations for which (R1) and (R2) cannot occur. The energy dependence apparently reflects this steric distinction. The preponderance of noncyclic configurations probably accounts for the predominance of (R3) seen at low collision energies. At higher collision energies (R3) declines rapidly and probably goes over to collisioninduced dissociation to form $\mathrm{Br}_{2}+\mathrm{Cl}_{2}+\mathrm{Cl}_{2}$, whereas (R1) increases and (R2) remains about constant. The corresponding reaction paths have been studied in the same way for the $\mathrm{HI}+\mathrm{Cl}_{2} \cdots \mathrm{Cl}_{2}$ system.

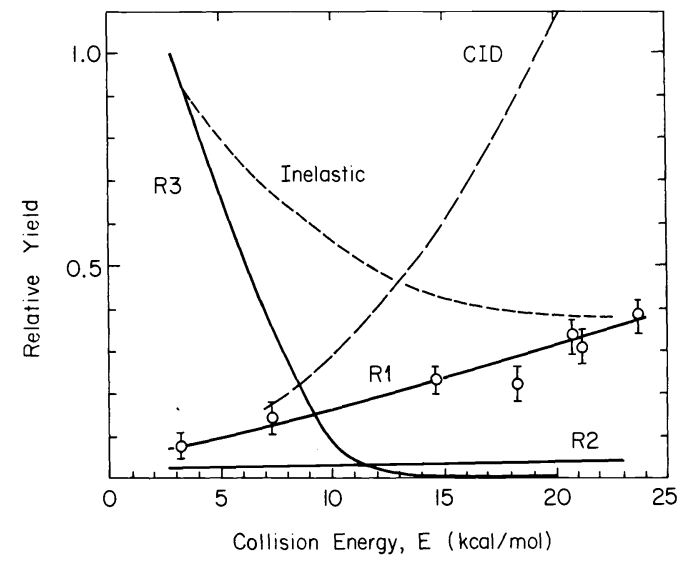

Fig. 12. Variation of yields with initial relative translational energy for five $\mathrm{Br}_{2}+\left(\mathrm{Cl}_{2}\right)_{2}$ collision processes: reactions producing (1) $\mathrm{BrCl}$, (2) $\mathrm{BrCl}_{3}$, and (3) $\mathrm{Br}_{2} \mathrm{Cl}_{2}$; inelastic scattering producing vibrationally excited $\left(\mathrm{Cl}_{2}\right)_{2}$; and collision induced dissociation (CID) producing $\mathrm{Br}_{2}+\mathrm{Cl}_{2}+\mathrm{Cl}_{2}$. Experimental points are included for reaction (1) to illustrate quality of the data. Absolute cross-section for reaction (3) estimated to be of the order $5-50 \AA^{2}$.

Figure 13 shows contour maps of the reactive scattering from (R1) for the $\mathrm{Br}_{2}$ and $\mathrm{HI}$ cases. The $\mathrm{BrCl}$ product peaks sharply forwards and backwards along the initial relative velocity vector whereas the ICI peaks backwards with respect to the incident $\mathrm{HI}$ direction. The $\mathrm{Cl}_{2}$ product distribution is broad for both reactions but in each case it peaks close in angle and velocity to the interhalogen product. The forward-backward symmetry of the reactive scattering for $\mathrm{Br}_{2}$ might be attributed either to statistical 

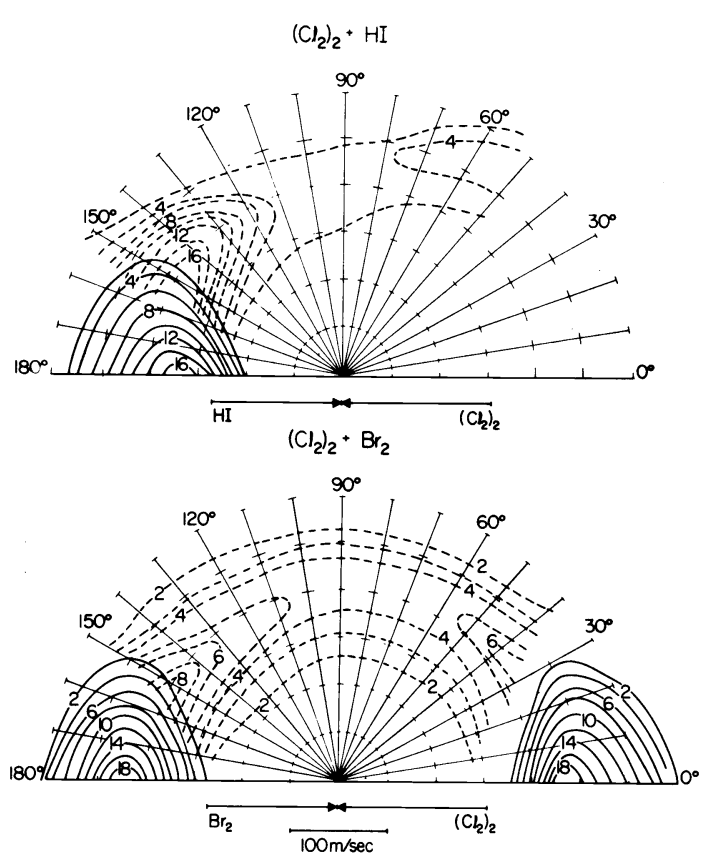

Fig. 13. Contour maps of product distributions from reactions of $\mathrm{Br}_{2}$ and $\mathrm{HI}$ with $\left(\mathrm{Cl}_{2}\right)_{2}$ at collision energies near $3 \mathrm{kcal} / \mathrm{mol}$. Full contours pertain to $\mathrm{BrCl}$ or $\mathrm{ICl}$ and dashed contours to $\mathrm{Cl}_{2}$ product.

dissociation of the collision complex or to recoil of the two $\mathrm{BrCl}$ molecules in opposite directions. The latter appears more likely, in view of the asymmetry found for the HI case. The experiments at higher collision energy give further evidence for the nonstatistical character of these reactions, both in the angular distributions and in the product translation.

Figure 14 outlines the reaction sequence inferred from the scattering data. ${ }^{55}$ The sharp peaking of the $\mathrm{BrCl}$ and $\mathrm{ICl}$ angular distributions shows these molecules are emitted with very high centrifugal momentum; the broader distribution of $\mathrm{Cl}_{2}$ shows it emerges with lower centrifugal momentum. These properties indicate a chain structure (perhaps quite nonlinear) for dissociative configurations of the reaction complex,

$$
X-\mathrm{Cl} \cdots \mathrm{Cl}-\mathrm{Cl} \cdots \mathrm{Cl}-Y \text {. }
$$

This presumably results from quick scission of the $X \cdots Y$ bond as the complex traverses the cyclic configuration. The nonstatistical character of the scattering indicates that at least one of the two weak $\mathrm{Cl} \cdots \mathrm{Cl}$ bonds also breaks quickly (within $\approx 10^{-12} \mathrm{sec}$ ). The comparable velocities found for the $\mathrm{Cl}_{2}$ and the $X-\mathrm{Cl}$ products suggest that the second $\mathrm{Cl} \cdots \mathrm{Cl}$ bond persists longer, at least until $X-$ $\mathrm{Cl} \cdots \mathrm{Cl}-\mathrm{Cl}$ and $\mathrm{Cl}-Y$ separate sufficiently to approach their asymptotic exit translational momenta (equal and opposite). For the $\mathrm{Br}_{2}$ reaction, the first $\mathrm{Cl} \cdots \mathrm{Cl}$ bond to break might be either one. For the $\mathrm{HI}$ case, the asymmetry of the $\mathrm{Cl}_{2}$ distribution indicates it is usually the bond which releases $\mathrm{HCl}$ and $\mathrm{ICl} \cdots \mathrm{Cl}_{2}$. There is some $\mathrm{Cl}_{2}$ corresponding to the opposte case, however, and this portion (at right in Fig. 13) shows higher velocities quantitatively consistent with the different mass distribution of the initial fratments ( $\mathrm{ICl}$ and $\left.\mathrm{HCl} \cdots \mathrm{Cl}_{2}\right)$. The (R2) path is a corollary of this sequence; it occurs instead of (R1) when the second $\mathrm{Cl} \cdots \mathrm{Cl}$ bond does not break.

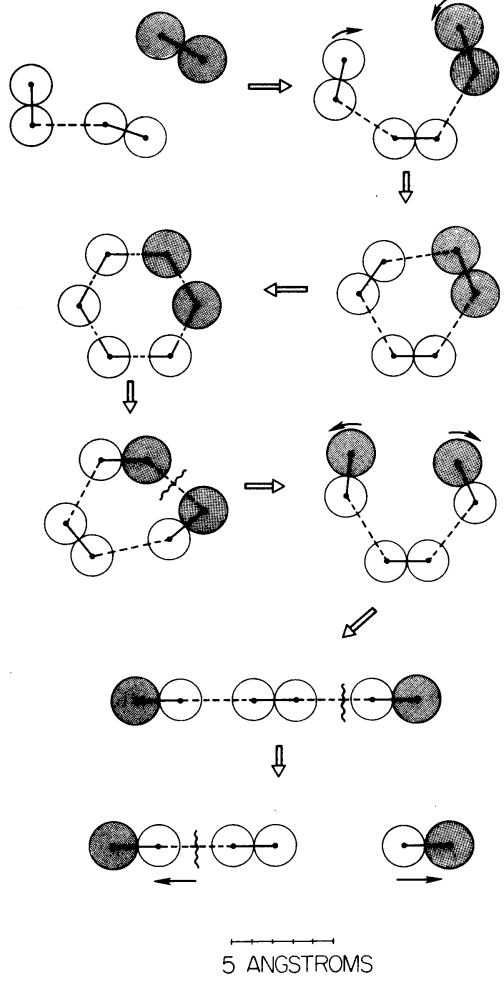

Fig. 14. Schematic reaction sequence for $\mathrm{Br}_{2}+\left(\mathrm{Cl}_{2}\right)_{2}$, illustrating the formation of van der Waals bonds (shown dashed), six center exchange, and three successive bond scissions.

Comparison of the angle-velocity distributions for (R2) and (R1) is quantitatively consistent with this interpretation. ${ }^{48}$ Several aspects of the data thus offer evidence that three sequential bond scissions can be resolved in these single collision experiments.

Reactive scattering for a host of other van der Waals molecules can be studied in the same way. Some reactions of dimeric hydrogen iodide and various organic molecules have been examined already. ${ }^{48}$ The van der Waals domain likewise poses new questions in collision dynamics and electronic theory. For instance, the drastic collisional energy transfer observed is probably due to the very weak and floppy bonding; the data now invite model calculations. It is interesting to find that the asymmetric scission of the two $\mathrm{Cl} \cdots \mathrm{Cl}$ bonds of the six-center reaction complex resembles that seen in photodissociation of $\mathrm{CH}_{3}$ $\mathrm{Cd}-\mathrm{CH}_{3}$ and other symmetric molecules. ${ }^{57}$ The electronic changes involved in switching between the extreme bond types will surely prove instructive. Merely adding an extra reagent molecule, linked by the very weak van der Waals bond, catalyzes what would otherwise be a forbidden chemical reaction.

Scattering and spectroscopic studies using much larger van der Waals polymers or molecular clusters are feasible, as such clusters are readily generated by increasing the source pressure behind a supersonic nozzle. For example, recent crossed-beam experiments ${ }^{58-60}$ find evidence that an incident atom or molecule will "stick" or "condense" on a sufficiently large polymer (typically with more than 20 atoms) to form an adduct which persists long enought to travel to the detector $\left(\approx 10^{-4} \mathrm{sec}\right)$. Figure 15 illustrates another theme. ${ }^{60}$ In the scattering of $\mathrm{HI}$ from ammonia polymers $\left(\mathrm{NH}_{3}\right)_{n}$, new mass peaks appear that arise from proton transfer with 

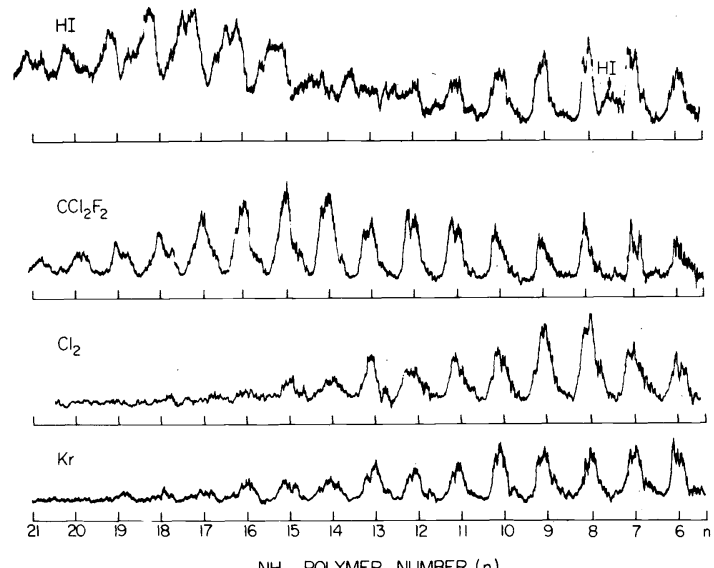

$\mathrm{NH}_{3}$ POLYMER NUMBER $(n)$

Fig. 15. Mass spectra (1\% resolution) of ammonia polymers $\left(\mathrm{NH}_{3}\right)_{n}$ with $n=6$ to 21 , scattered at $10^{\circ}$ from the ammonia beam by collisions with $\mathrm{Kr}, \mathrm{Cl}_{2}, \mathrm{CCl}_{2} \mathrm{~F}_{2}$, and $\mathrm{HI}$. For the $\mathrm{HI}$ case additional peaks are seen which correspond to $\mathrm{NH}_{4}{ }^{+} \mathrm{I}^{-}\left(\mathrm{NH}_{3}\right)_{m}$ with $m=2$ to 13 .

solvation of the resulting salt molecule,

$$
\mathrm{HI}+\left(\mathrm{NH}_{3}\right)_{n} \rightarrow\left(\mathrm{NH}_{4}^{+} \mathrm{I}^{-}\right)\left(\mathrm{NH}_{3}\right)_{m}+\left(\mathrm{NH}_{3}\right)_{n-m-1} \text {. }
$$

In such experiments the polymer numbers $n$ and $m$ can be scanned by adjusting the beam source conditions. An interesting possibility now being explored is the use of such beams with laser-induced fluoresence to study solvation. The emission from the solvated solute molecule would show how its vibrational force constant and bond distance changes with the number of solvent molecules. This would almost amount to watching the solute dissolve.

The vigor and spirit with which experiments and theory in quest of "single collision chemistry" are now being pursued in many laboratories is reminiscent of the opening up of molecular spectroscopy and structure forty years ago. The results already obtained and the opportunities defined suggest a comparable development of basic facts and insight into reaction dynamics can be expected. This will apply to chemical phenomena far beyond the realm of collision chambers and computers.

Acknowledgements-It is a pleasure to thank my students and colleagues devoted to the study of reaction dynamics and to acknowledge gratefully support of the work at Harvard by the National Science Foundation.

\section{REFERENCES}

${ }^{1}$ R. D. Levine and R. B. Bernstein, Molecular Reaction Dynamics. Clarendon Press, Oxford (1974).

${ }^{2}$ M. A. D. Fluendy and K. P. Lawley, Chemical Applications of Molecular Beam Scattering. Chapman \& Hall, London (1973). ${ }^{3}$ D. R. Herschbach, Faraday Discuss. Chem. Soc. 55, 233 (1973).

${ }^{4}$ J. M. Farrar and Y. T. Lee, Ann. Rev. Phys. Chem. 25, 357 (1974); J. P. Toennies, in Physical Chemistry-An Advanced Treatise. (eds. H. Eyring, W. Jost and D. Henderson) Vol. VIA, Academic Press, New York (1975); R. Grice, Adv. Chem. Phys. 30, 247 (1975).

${ }^{5}$ R. A. Marcus, Faraday Discuss. Chem. Soc. 55, 9 (1973).

${ }^{6}$ R. D. Levine and R. B. Bernstein, Accts. Chem. Res. 7, 393 (1974).

${ }^{7}$ J. C. Polanyi, Faraday Discuss. Chem. Soc. 55, 389 (1973); J. C. Polanyi and J. L. Schreiber, in Physical Chemistry-An Advanced Treatise. (eds. H. Eyring, W. Jost and D. Henderson) Vol. 6a, p. 460. Academic Press, new York (1974).
${ }^{8}$ R. N. Porter, Ann. Revs. Phys. Chem. 25, 317 (1974).

${ }^{9}$ B. H. Mahan, Accts. Chem. Res. 1, 217 (1968); 3, 393 (1970); 8, 55 (1975); Z. Herman and R. Wolfgang, in Ion-Molecule Reactions. (ed. J. L. Franklin) Plenum Press, New York (1972)

${ }^{10}$ J. D. McDonald, P. R. LeBreton, Y. T. Lee and D. R. Herschbach, J. Chem. Phys. 56, 769 (1972).

${ }^{11}$ J. B. Anderson, R. P. Andres and J. B. Fenn, Adv. Chem. Phys. 10, 275 (1966); J. B. Anderson in Molecular Beams and Low Density Gas Dynamics. (ed. P. P. Wegener) Vol. IV, Marcel Dekker, New York (1974).

${ }^{12}$ R. A. Larsen, S. K. Neoh and D. R. Herschbach, Rev. Sci. Instrum. 45, 1511 (1974).

${ }^{13}$ J. E. Mosch, S. A. Safron and J. P. Toennies, Chem. Phys. 8, 304 (1975) and work cited therein.

${ }^{14}$ P. R. Brooks, Faraday Discuss. Chem. Soc. 55, 299 (1973) and work cited therein.

${ }^{15}$ D. S. Y. Hsu, G. M. McClelland and D. R. Herschbach, J. Chem. Phys. 61, 4927 (1974).

${ }^{16}$ R. P. Mariella, D. R. Herschbach and W. Klemperer, J. Chem. Phys. 58, 3785 (1973).

${ }^{17}$ T. Carrington and J. C. Polanyi, MTP Int. Rev. Sci., Phys. Chem., Ser. One, 9, 135 (1972).

${ }^{18} \mathrm{~J}$. G. Moehlmann and J. D. McDonald, J. Chem. Phys. 62, 3052, 3061 (1975).

${ }^{19}$ S. J. Riley and K. R. Wilson, Faraday Discuss. Chem. Soc. 53 132 (1972); R. Bersohn, J. Chem. Phys. 63, 809 (1975).

${ }^{20}$ T. J. Odiorne, P. R. Brooks and J. V. V. Kasper, J. Chem. Phys. 55, 1980 (1971).

${ }^{21}$ R. N. Zare and P. J. Dagdigian, Science 185, 739 (1974).

${ }^{22}$ D. A. Case and D. R. Herschbach, Mol. Phys. 30, 1537 (1975).

${ }^{23}$ N. H. Hijazi and J. C. Polanyi, J. Chem. Phys. 63, 2249 (1975)

${ }^{24} \mathrm{~J}$. Grosser and H. Haberland, Chem. Phys. 2, 342 (1973).

${ }^{25}$ M. A. Nazar, J. C. Polanyi and W. J. Skrlac, Chem. Phys. Letters 29, 473 (1974).

${ }^{26}$ U. Dinur and R. D. Levine, Chem. Phys. Letters 31, 410 (1975).

${ }^{27}$ D. R. Herschbach, in Potential Energy Surfaces in Chemistry. (ed. W. A. Lester) p. 44. IBM Laboratory, San Jose (1971)

${ }^{28}$ A. D. Walsh, J. Chem. Soc. 1953, 2266 (1953); R. J. Buenker and S. D. Peyerimhoff, Chem. Revs. 74, 127 (1974).

${ }^{29}$ G. H. Kwei and D. R. Herschbach, J. Chem. Phys. 51, 1742 (1969).

${ }^{30}$ B. S. Rabinovitch and D. W. Setser, Advan. Photochem. 3, 1 (1964).

${ }^{31}$ J. T. Cheung, J. D. McDonald and D. R. Herschbach, J. Am. Chem. Soc. 95, 7889 (1973).

${ }^{32} \mathrm{O}$. K. Rice, Statistical Mechanics, Thermodynamics, and Kinetics. pp. 495-573. W. A. Freeman, San Francisco, (1967); R. A. Marcus, J. Chem. Phys. 43, 2658 (1965).

${ }^{33}$ S. A. Safron, N. D. Weinstein, D. R. Herschbach and J. C. Tully, Chem. Phys. Lett. 12, 564 (1972). See also, R. A. Marcus, J. Chem. Phys. 62, 5015 (1975)

${ }^{34}$ W. B. Miller, S. A. Safron and D. R. Herschbach, Faraday Discuss. 44, 108, 292 (1967); J. Chem. Phys. 56, 3581 (1972).

${ }^{35}$ D. D. Parrish and D. R. Herschbach, J. Am. Chem. Soc. 95, 6133 (1973); D. St. A. G. Radlein, J. C. Whitehead and R. Grice, Mol. Phys. 29, 1813 (1975).

${ }^{36}$ S. J. Riley and D. R. Herschbach, J. Chem. Phys. 58, 27 (1973).

${ }^{37}$ G. A. Fisk, J. D. McDonald and D. R. Herschbach, Disc. Faraday Soc. 44, 228 (1967); M. K. Bullit, C. H. Fisher and J. L. Kinsey, J. Chem. Phys. 60, 5209 (1974).

${ }^{38}$ R. M. Noyes, J. Am. Chem. Soc. 70, 2614 (1968)

${ }^{39}$ J. F. Harris, J. Am. Chem. Soc. 84, 3148 (1962); F. S. Dainton and B. E. Fleischfressen, Trans. Faraday Soc. 62, 1838 (1966).

${ }^{40}$ Y. T. Lee, Ber. Bunsen. physik. Chem. 78, 135 (1974).

${ }^{41}$ G. H. Kwei, A. B. Lees and J. A. Silver, J. Chem. Phys. 58, 1710 (1973); G. Aniansson, R. P. Creaser, W. D. Held and J. P. Toennies, J. Chem. Phys. 61, 5381 (1974); S. Stolte, A. E. Proctor and R. B. Bernstein; J. Chem. Phys. 61, 3855 (1974); 62, 2506 (1975).

${ }^{42}$ A. C. Roach and M. S. Child, Mol. Phys. 14, 1 (1968); W. S. Struve, Mol. Phys. 25, 777 (1973).

${ }^{43}$ R. Hoffmann, J. Chem. Phys. 49, 3740 (1969); L. C. Cusacha, M. Krieger and C. W. McCurdy, Int. J. Quantum Chemistry 35, 67 (1969); R. N. Porter and L. M. Raff, J. Chem. Phys. 50, 5216; 51, 
1623 (1969); B. M. Gimarc, J. Chem. Phys. 53, 1623 (1970); W. A. Goddard, J. Am. Chem. Soc. 94, 793 (1972).

${ }^{44}$ D. M. Silver and R. M. Stevens, J. Chem. Phys. 59, 3378 (1973) and work cited therein.

${ }^{45}$ S. H. Bauer and E. Ossa, J. Chem. Phys. 45, 434 (1966); A. Burcat and A. Lifschitz, J. Chem. Phys. 47, 3079 (1967); R. D. Kern and G. G. Nika, J. Phys. Chem. 75, 1015 (1971).

${ }^{46}$ S. H. Bauer, D. M. Lederman, E. L. Resler and E. R. Fisher, Int. J. Chem. Kin. 5, 93 (1973).

${ }^{47}$ J. S. Wright, Chem. Phys. Letters 6, 476 (1970); Can. J. Chem. 53, 549 (1975).

${ }^{48}$ D. A. Dixon, Ph.D. Thesis, Harvard University (1975).

${ }^{49}$ J. H. Sullivan, J. Chem. Phys. 46, 73 (1967).

${ }^{50}$ P. Schweitzer and R. M. Noyes, J. Amer. Chem. Soc. 93, 3561 (1971).

${ }^{51}$ S. B. Jaffe and F. B. Anderson, J. Chem. Phys. 51, 1057 (1969).

${ }^{52}$ D. L. King and D. R. Herschbach, Faraday Discuss. Chem. Soc. 55, 331 (1973) and work cited therein.
${ }^{53}$ P. Brumer and M. Karplus, Faraday Discuss. Chem. Soc. 55, 80 (1973).

${ }^{54}$ S. W. Benson and G. R. Haugen, J. Amer. Chem. Soc. 87, 4936 (1965); A. Maccoll, Chem. Rev. 69, 33 (1969).

${ }^{55}$ D. L. King, D. A. Dixon and D. R. Herschbach, J. Am. Chem. Soc. 96, 3328 (1974); D. A. Dixon and D. R. Herschbach, J. Am. Chem. Soc. in press (1975).

${ }^{56}$ S. J. Harris, S. E. Novick, J. S. Winn and W. Klemperer, J. Chem. Phys. 61, 3866 (1974).

${ }^{57}$ M. Tamir, U. Halavee and R. D. Levine, Chem. Phys. Letters 25, 38 (1974).

${ }^{58}$ A. G. Urena, R. B. Bernstein and G. R. Phillips, J. Chem. Phys. 62, 1818 (1975).

${ }^{59}$ R. Behrens, A. Freedman, R. R. Herm and T. P. Parr, J. Chem. Phys. 63, 4622 (1975).

${ }^{60}$ J. T. Cheung, D. A. Dixon and D. R. Herschbach, J. Am. Chem. Soc. To be published. 